\title{
Uso do gel da camomila (Matricaria chamomilla l.) associado ao LED vermelho de baixa frequência no tratamento da acne vulgar
}

\author{
Use of chamomile gel (Matricaria chamomilla l.) associated with low frequency red LED in the \\ treatment of vulgar acne \\ Uso de gel de manzanilla (Matricaria chamomilla l.) asociado con LED rojo de baja frecuencia en el \\ tratamiento del acné vulgar
}

Recebido: 01/11/2021 | Revisado: 09/11/2021 | Aceito: 13/11/2021 | Publicado: 23/11/2021

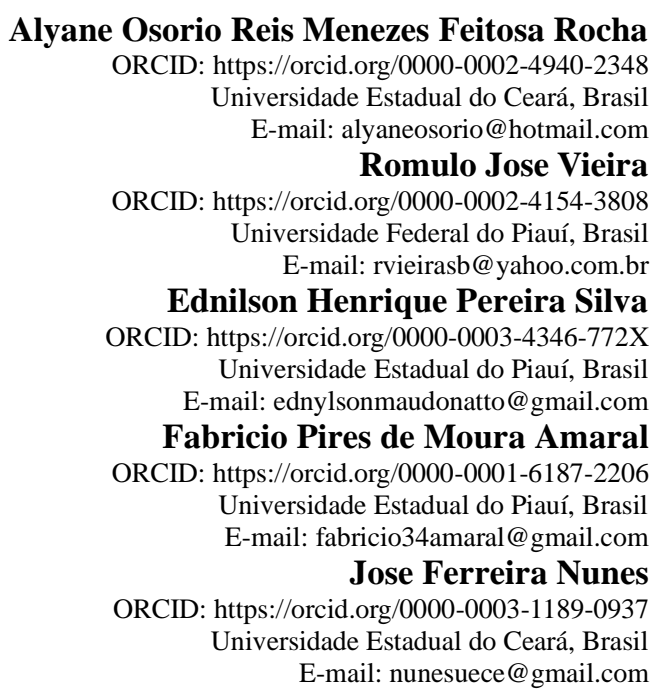

\begin{abstract}
Resumo
A acne vulgar é uma dermatose crônica do folículo pilossebáceo, que possui, como fatores fundamentais, hiperprodução sebácea. Não existe perfil epidemiológico universal para a mesma. Atinge ambos os gêneros e é um problema de saúde pública. O objetivo deste trabalho foi avaliar a eficácia do tratamento do gel da Camomila (Matricaria chamomilla L.) associado ao LED Vermelho de baixa frequência $(4,82-3,84 \mathrm{~Hz})$ no tratamento da Acne Vulgar. Trata-se de um estudo de campo, experimental com abordagem quanti - qualitativa incluindo 20 pacientes do gênero feminino atendidas em uma clínica de Fisioterapia Dermatofuncional da Cidade de Floriano-PI. As pacientes foram alocadas em grupos de cinco mulheres cada. Para avaliação do estado da pele foram utilizadas a Escala Visual de Percepção facial - EVPF e a avaliação por Contagem no Número de Lesão - CNL. Ao fim do estudo constatou-se que o tratamento da acne com uso do gel da camomila a 5\% associada ao aparelho de LED Vermelho de baixa frequência $(4,82-3,84 \mathrm{~Hz})$, mostrou-se efetivo na diminuição do processo inflamatório da pele. Apresentando-se como uma terapêutica segura, acessível e eficaz. Sendo também importante outros cuidados especiais com a pele nos pacientes com essa afecção dermatológica.
\end{abstract}

Palavras-chave: Acne; LED Vermelho; Fototerapia; Tratamento da acne.

\begin{abstract}
Acne vulgaris is a chronic dermatosis of the pilosebaceous follicle, which has, as fundamental factors, sebaceous hyperproduction. There is no universal epidemiological profile for it. It affects both genders and is a public health problem. The objective of this work was to evaluate the effectiveness of the treatment of Chamomile gel (Matricaria chamomilla L.) associated with low frequency Red LED $(4.82-3.84 \mathrm{~Hz})$ in the treatment of Acne Vulgaris. This is an experimental field study with a quanti-qualitative approach including 20 female patients seen at a Dermatofunctional Physical Therapy clinic in the city of Floriano-PI. Patients were divided into groups of five women each. To assess the condition of the skin, the Visual Facial Perception Scale - EVPF and the assessment by Counting the Number of Lesions - CNL were used. At the end of the study, it was found that the treatment of acne using 5\% chamomile gel associated with a low-frequency Red LED device $(4.82-3.84 \mathrm{~Hz})$, proved to be effective in reducing the inflammatory process of the skin. Presenting itself as a safe, accessible and effective therapy. Other special skin care is also important for patients with this dermatological condition.
\end{abstract}

Keywords: Acne; Red LED; Phototherapy; Acne treatment. 


\begin{abstract}
Resumen
El acné vulgar es una dermatosis crónica del folículo pilosebáceo, que tiene como factores fundamentales la hiperproducción sebácea. No existe un perfil epidemiológico universal para ello. Afecta a ambos sexos y es un problema de salud pública. El objetivo de este trabajo fue evaluar la efectividad del tratamiento del gel de Manzanilla (Matricaria chamomilla L.) asociado a LED Rojo de baja frecuencia $(4.82-3.84 \mathrm{~Hz})$ en el tratamiento del Acné Vulgaris. Se trata de un estudio de campo experimental con un enfoque cuanti-cualitativo que incluyó a 20 pacientes atendidas en una clínica de fisioterapia dermatofuncional en la ciudad de Floriano-PI. Los pacientes se dividieron en grupos de cinco mujeres cada uno. Para evaluar el estado de la piel se utilizó la Escala de Percepción Visual Facial EVPF y la evaluación por Conteo del Número de Lesiones - CNL. Al final del estudio, se encontró que el tratamiento del acné con gel de manzanilla al 5\% asociado con un dispositivo LED rojo de baja frecuencia $(4.82-3.84 \mathrm{~Hz})$, demostró ser efectivo para reducir el proceso inflamatorio de la piel. Presentándose como una terapia segura, accesible y eficaz. Otro cuidado especial de la piel también es importante para los pacientes con esta afección dermatológica.
\end{abstract}

Palabras clave: Acné; LED rojo; Fototerapia; Tratamiento contra el acné.

\title{
1. Introdução
}

A acne é a mais comum das doenças crônicas do folículo pilossebáceo da pele humana, causada por inúmeros fatores e que leva ao aparecimento de vários tipos de lesão (Ribeiro, 2010). Segundo Rebello (2005) o uso da Camomila (Matricaria chamomilla L.) tem a finalidade de eliminar, ou pelo menos atenuar, o estado inflamatório da pele, diminuindo o eritema.

A pele contém muitos folículos pilosos diminutos, ou poros. Cada poro contém um pelo e uma glândula multilobulada chamada glândula sebácea. As glândulas sebáceas produzem uma substância oleosa chamado sebo que normalmente se desloca até o poro para lubrificar o pelo e a pele (Manual MSD: Merck Sharp \& Dohme, 2020).

Existe evidência que a aplicação do Laser de baixa frequência estimula diferentes processos do metabolismo celular com resultados satisfatórios na melhora da flacidez tissular, na prevenção do envelhecimento precoce, na eliminação de edemas, na melhora da cicatrização em processos pós-cirúrgicos, na estimulação dos folículos pilosos nos tratamentos de terapia capilar, no clareamento de manchas e especialmente nos tratamentos de acne (Oliveira, et al. 2014). Desse modo, a utilização concomitante dessas duas terapêuticas pode potencializar os resultados obtidos por estes procedimentos quando utilizados isoladamente.

Camomila ou sinônimo Matricaria recutita (L.) Rauschert é uma popular erva medicinal aromática usada principalmente para fins terapêuticos. Flores secas e óleo essencial são os produtos mais amplamente usados, principalmente porque têm múltiplas propriedades medicinais, desde antiinflamatórias, analgésicas, antimicrobianas e antiespasmicas a sedativa (Gardiner, 2007). No Brasil, foi introduzida pelos imigrantes europeus há mais de 100 anos. Atualmente, é a planta medicinal com a maior área de cultivo no território brasileiro (Mckay \& Blumberg, 2006).

Analisando a atividade terapêutica da camomila, Alonso (2004) afirmou que a mesma, é determinada pelos princípios ativos lipofilicos e pelos hidrofílicos, a atividade predominante do extrato aquoso é espasmolitica, enquanto o estratoalcoólico apresentou uma atividade antiflogística e que o camazuleno possui reconhecida atividade antiinflamatória, que é reforçada pela presença de matricina e alfa bisabolol.

A fototerapia de baixa intensidade utiliza fontes de luz LASER (Light Amplification by Stimulated Emission of Radiation) ou LED (Light Emitting Diodes), em comprimentos de onda que podem envolver desde os da luz visível ao infravermelho (600-1000 nm) (Carroll et al. 2014; Huang et al. 2009; Peplow et al. 2011). Nessa terapia, a fonte de luz é aplicada sobre uma área determinada, utilizando-se baixos valores de densidade de potência $(\mathrm{mW} / \mathrm{cm} 2)$ e doses de energia (J/cm2 ). Desse modo, não são observados efeitos térmicos danosos à região de aplicação (Carroll et al. 2014; HUANG et al. 2009). 
Dentro do contexto utilizado para a pesquisa, o trabalho identificou o seguinte problema: Quais os efeitos da Camomila (Matricaria chamomilla L.) e sua associação ao LED Vermelho de baixa frequência $(4,82-3,84 \mathrm{~Hz})$ no tratamento da Acne Vulgar? Por assim dizer, este estudo se mostra extremamente relevante pois visa contribuir diretamente com o tratamento dermatológico usado na acne vulvar.

\section{Método}

Trata-se de um estudo de campo, experimental com abordagem quanti qualitativa e natureza aplicada realizado numa Clínica de Fisioterapia Dermatofuncional que atende através da rede particular, sendo a mesma especializada em estética facial e corporal na cidade de Floriano - Piauí. O estudo foi realizado no período de novembro/dezembro de 2020 e janeiro/fevereiro/março de 2021. Aprovado sobre o parecer de número: 4.270.867.

A população alvo do estudo foram pacientes atendidas nessa Clínica. A amostra foi sistemática e constou de 20 pacientes do gênero feminino com características clínicas de acne inflamatória, sendo excluído pacientes do gênero masculino e pacientes que não apresentavam acne ativa. Os critérios de inclusão foram pacientes atendidos pela clínica há mais de um mês e elegíveis para o estudo em questão.

\section{Procedimentos Clínicos da Execução do Estudo}

As pacientes que atenderam aos critérios de inclusão, foram divididas aleatoriamente em quatro grupos nomeados G1, G2, G3, G4. No primeiro grupo (G1) utilizou-se o gel da camomila a 5\%, no Segundo grupo (G2) utilizouse o aparelho de LED Vermelho, no Terceiro grupo (G3) utilizou-se o gel da camomila a 5\% associado ao aparelho de LED Vermelho e no quarto grupo (G4) utilizou-se o tratamento padrão (uso de uma loção secativa a base de acneol + ácido lactobiônico + TGP2 peptídeo) realizado de rotina na clínica. A duração de aplicação de cada procedimento por grupo foram de $20 \mathrm{~min}$. Realizou-se a fotodocumentção do antes do procedimento terapêutico e do depois após quatro dias seguidos do procedimento terapêutico, em cada grupo, como avaliação final.

O procedimento aconteceu no consultório da Clínica mencionada. Para higienização utilizou-se álcool a 70\% e em seguida aplicava-se o procedimento do referido grupo. As aplicações foram realizadas durante quatro dias seguidos para cada paciente de cada grupo.

Foi utilizado o Extrato glicólico não iônico da Camomila (Matricaria chamomilla L.) à base de $5 \%$ em gel que foi manipulado em uma Farmácia de manipulação da Cidade de Floriano-PI. A base do gel à 5\% deu-se após uma pesquisa em dados de Patentes e por ser utilizada no tratamento da acne nessa Farmácia de manipulação.

O aparelho de LED Vermelho utilizado foi uma câmera LED portátil Facial e Corporal da marca Prumemedhomeuse composta de 287 leds, voltagem de 110- 240V, frequência de 50-60Hz, voltagem de saída de $12 \mathrm{~V}$ e potência de 10W. A luz led vermelha apresenta comprimento de onda (620-750nm).

\section{Resultados e Discussões}

Os resultados são apresentados a seguir, segundo os grupos (G1, G2, G3 e G4). As participantes dos quatro grupos realizaram as aplicações nos dias estipulados e nenhuma apresentou intercorrência. Foram avaliadas com relação ao tipo de acne mediante teste semiológico e clínico da face e das respostas do questionário aplicado, conforme os gráficos a seguir. O questionário era composto de 18 questões, sendo selecionadas algumas perguntas e respostas para a discussão. 
Gráfico 1: Caracterização do tipo de acne apresentado pelas pacientes deste estudo. Floriano-PI, 2021.

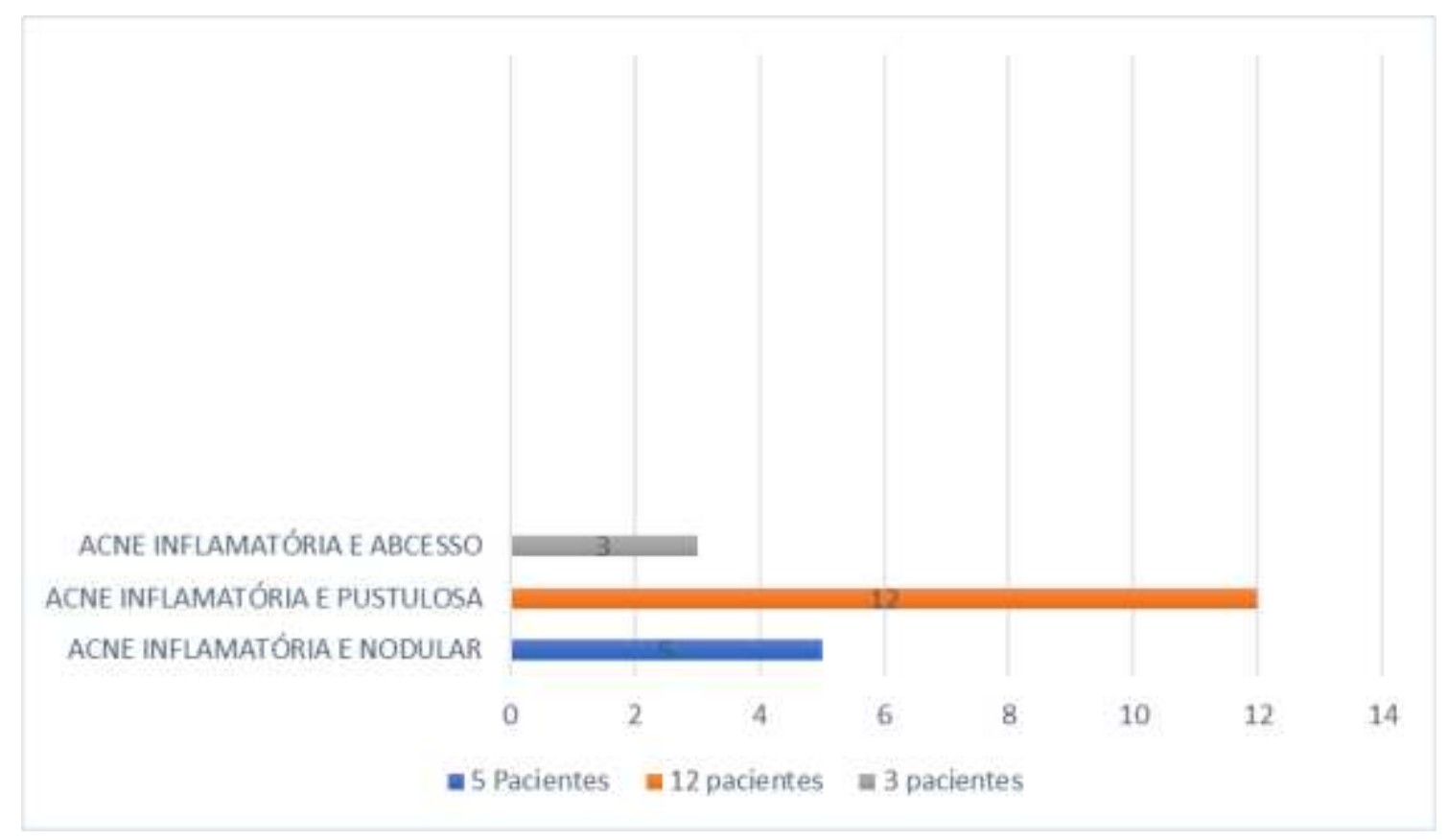

Fonte: Autores (2021).

Após avaliação das 20 pacientes, cinco delas apresentavam acne inflamatória nodular, 12 destas tinham acne inflamatória e pustulosa, e somente três pacientes apresentaram acne inflamatória e abcesso. É necessário afirmar que o experimento constou com a análise de 20 mulheres com presença de acne ativa na região facial. Das mulheres atendidas, 15 delas não realizaram nenhum tratamento estético anterior e 18 pacientes não utilizaram nenhum tipo de sabonete com ácido, o que não interferia no tratamento.

De acordo com os grupos atendidos, muitas delas queixavam-se de inflamações recorrentes, um dos motivos que fizeram elas participarem dessa pesquisa. É importante salientar que as mesmas foram informadas sobre a importância dos cuidados com a pele, ressaltando a eficácia do tratamento proposto, uma mais nova alternativa para o tratamento da acne foi o uso do gel de camomila a 5\% associado ao LED Vermelho de baixa frequência.

Para Michalun (2010) as condições da pele com acne caracterizam-se por pequenos pontos de infecção, numerosos ou esparsos. A pele que apresenta acne é oleosa e, em muitos casos pode aparecer cravos, mílios e poros entupidos. Os produtos mais eficazes são aqueles com ingredientes que regulam a secreção de glândulas sebáceas, hidratam, curam, corrige o pH da pele, aliviam e reduzem a inflamação, apresentam ação antibacteriana e promovem uma leve esfoliação das camadas corneócitas. Partindo do contexto, os grupos G1(utilizou o gel da Camomila a 5\%) e G3 (utilizou o gel da camomila a 5\% associado ao LED Vermelho de baixa frequência) utilizaram assim um produto elaborado afim de reduzir a inflamação como sugeriu Michalun (2010). Sendo que o grupo que também utilizou o Led vermelho de forma associada que foi o grupo (G3) obteve melhora significativa na inflamação. 
Gráfico 2: Região de apresentação da acne ativa dos grupos (G1-G2-G3-G4). Floriano-PI, 2021.

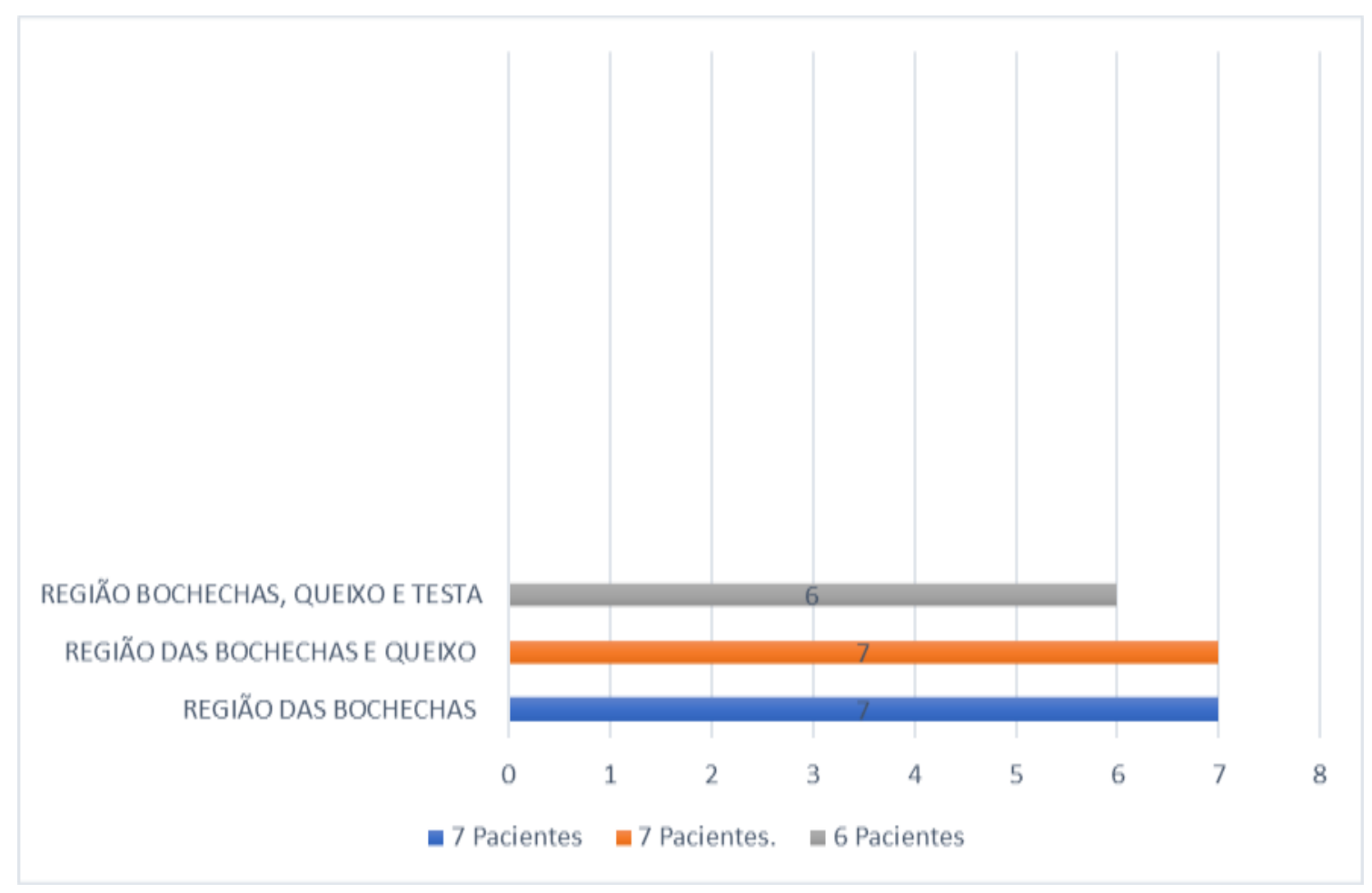

Fonte: Autores (2021).

Os resultados apontam que sete pacientes possuíam acne localizada na região da bochecha e outras sete na região entre bochecha e queixo. Apenas seis dessas pacientes apresentavam uma maior proporção de acne, localizadas nas regiões que contemplavam as bochechas, queixo e testa. Observações também mencionadas por Mcginley (1980) apud Matarazzo (2016), quando se trata da acne, a população de Propionibacterium acnes é maior na face e no tronco superior, locais com maior concentração lipídica, mostrando, aí, relação direta entre população bacteriana e seborréia local. Saliências, tais como cravos, comedões, espinhas, cistos, e, às vezes, abscessos, se formam na pele, geralmente na face, no tórax, nos ombros ou nas costas.

As pacientes dos quatros grupos realizavam extração em casa, por ser regiões de fácil acesso e ainda aplicavam produtos aleatórios, sem indicação de um profissional, prejudicando mais ainda as regiões já afetadas, proliferando a bactéria e aumentando a inflamação por toda a face. Sendo observado que em todos os grupos atendidos, as acnes eram recorrentes e inflamatórias.

De acordo com a Sociedade Brasileira de Dermatologia - SBD (2018) a chamada zona "T" localiza-se entre testa, nariz e queixo, e se caracteriza como sendo a maior parte do rosto com maior oleosidade. Nos quatro grupos do experimento, as regiões mais evidenciadas com oleosidade e com inflamação foram as bochechas e o queixo.

A maioria das pacientes relatava não fazer controle da dieta alimentar. Outro ponto importante para aumentar a oleosidade da pele, aumentar a acne e dificultar o tratamento. Uma alimentação hipercalórica influencia na inflamação da pele, dificultando os tipos de tratamento pré-existentes no mercado. Durante a avaliação inicial é um requisito muito importante a ser questionado. 
Gráfico 3: Avaliação inicial dos grupos (G1-G2-G3-G4) de acordo com a Escala Visual de Percepção Facial - EVPF. Floriano-PI, 2021.

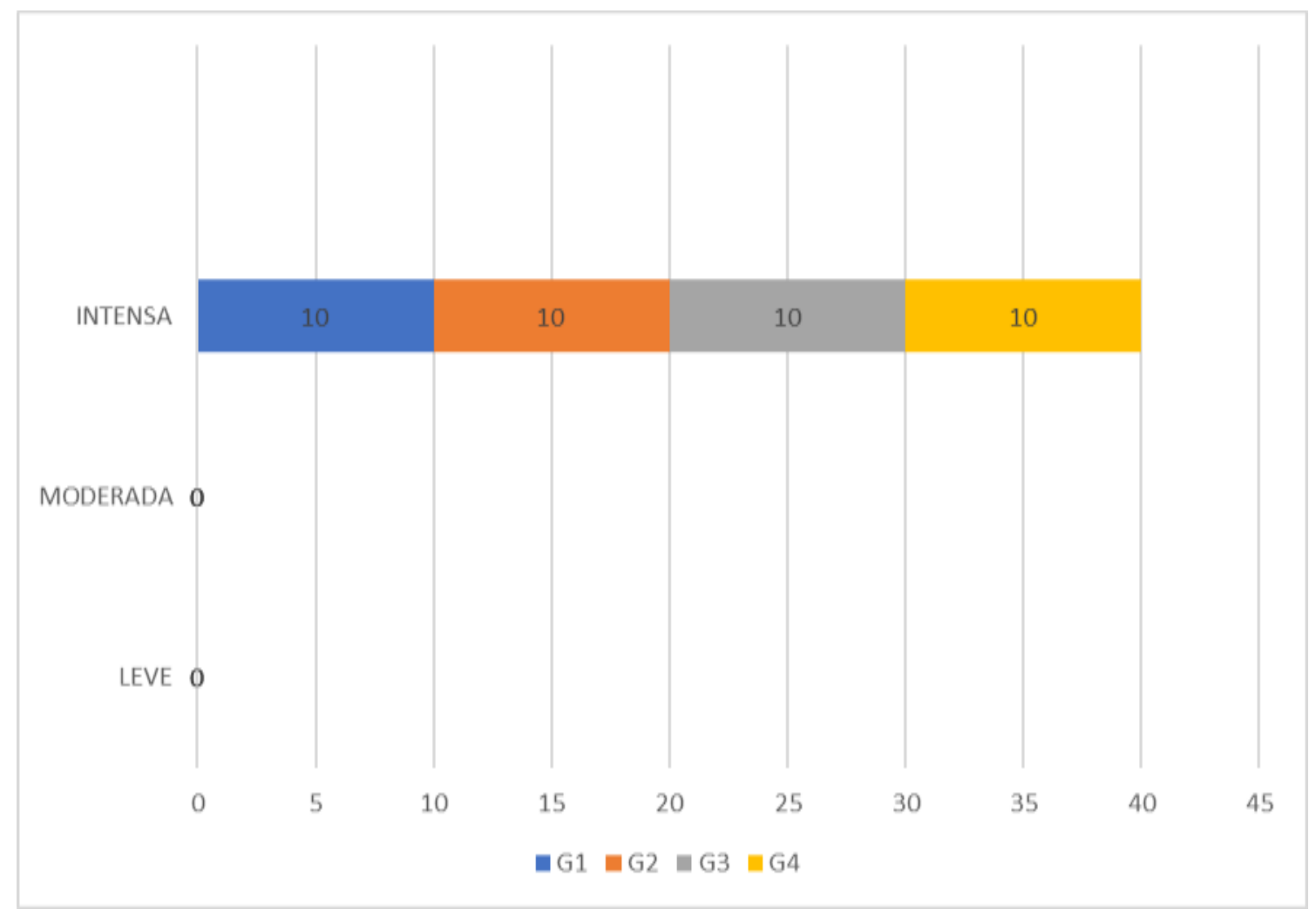

Fonte: Autores (2021).

Para Avaliação Inicial da face das participantes foi utilizada a Escala Visual de Percepção Facial - EVPF aplicada em dois momentos na $1^{a}$ sessão (avaliação inicial) e na $4^{a}$ sessão (avaliação final). Essa escala foi dividia em Leve (0-2), Moderada (3-7) e intensa (8-10). Nos grupos atendidos (G1-G2-G3-G4) de acordo com a classificação correspondeu a Intensa (intensidade 8 na escala). Quanto maior pontuação maior insatisfação e quanto menor pontuação maior satisfação facial.

$\mathrm{Na}$ análise da estrutura da pele, as pacientes atendidas mostravam-se insatisfeita com a face. A estatística descritiva mostrou que $100 \%$ das mulheres atendidas nos quatro grupos possuíam acne ativa, inflamada e de classificação intensa EVPF. A Escala de Avaliação da Percepção Visual foi fundamental para analisar a percepção das pacientes quanto ao grau da acne. Em um estudo semelhante ao da presente pesquisa, Yamada e Silva (2017) avaliaram 10 voluntários com idade 21,5 $\pm 1,4$ anos que apresentavam acne grau 2 e 3.

A Escala Visual de Percepção Facial (EVPF) foi aplicada em três momentos, no início, ao longo do tratamento (meio) e no final do tratamento para avaliação do grau de satisfação facial. Em se tratando dos tratamentos estético anteriores analisados no Grupo (03) que utilizou o gel a 5\% da camomila associado ao LED, 60\% das pacientes não realizavam nenhum procedimento estético anterior ao estudo, sendo este seu primeiro e $40 \%$ já realizaram procedimentos anteriores a este.

De acordo com Silveira (2018) o tratamento da acne vulgar pelo profissional de dermatologia e estética tem a finalidade de manter a pele limpa e saudável, livre de comedões e pústulas, inibir a proliferação bacteriana e a inflamação e prevenir a formação de cicatrizes inestéticas, dentre outros motivos. Uma das consequências de não tratar a acne é o aparecimento de cicatriz, que se forma como resultado de um processo inflamatório constante. A cicatriz também é uma queixa dos pacientes que possuem acne vulgar. Dessa forma observou-se que a maioria não realizava tratamentos anteriores e algumas já apresentavam cicatrizes da acne. 
O que se observou no presente estudo é que as pacientes do grupo (G3) não sofreram influência do ácido salicílico no tratamento proposto. Na prática clínica quando o uso constante desse produto na região facial pode afinar as camadas da pele e provocar aumento da quantidade de acne. Assim pôde-se observar também a ação do gel de camomila a 5\% ativado com a ação do LED Vermelho de comprimento de onda $(620-750 \mathrm{~nm})$ foi ideal para provocar um efeito calmante e anti-inflamatório na pele. Nesse caso trata-se o processo inflamatório sem agredir a pele.

É importante salientar que os grupos (G2) e (G3) utilizaram o LED Vermelho no seu procedimento e especificamente em se tratando do grupo (G3) que com ação da camomila em gel a 5\% acelerou a diminuição da inflamação provocada pela acne. Em um modelo de retalho de pele em ratos Dungel et al. (2014) confirmaram que a luz LED Vermelha induziu efeitos pró-angiogênicos, na proliferação e regeneração tecidual. Esse efeito regenerador foi observada em evidencia no grupo (G3), com os pacientes durante a avaliação final, onde já apresentavam resultados positivos no quadro inflamatório.

Compreende-se assim, conforme Hernandéz (2018) que os macrófagos expostos a comprimento de onda em torno $(660 \mathrm{~nm})$ liberam citoquinas que estimulam a proliferação de fibroblastos e a produção de fatores de crescimento, influenciando o processo de cicatrização e reparo de feridas. Confirmando a observação desse autor, nesse experimento as pacientes que utilizaram o gel de camomila a 5\% e o LED Vermelho com comprimentos de ondas de (620-750nm), apresentaram melhoria no reparo das lesões inflamatórias da acne.

Figura 1: Paciente 1 do Grupo 1.
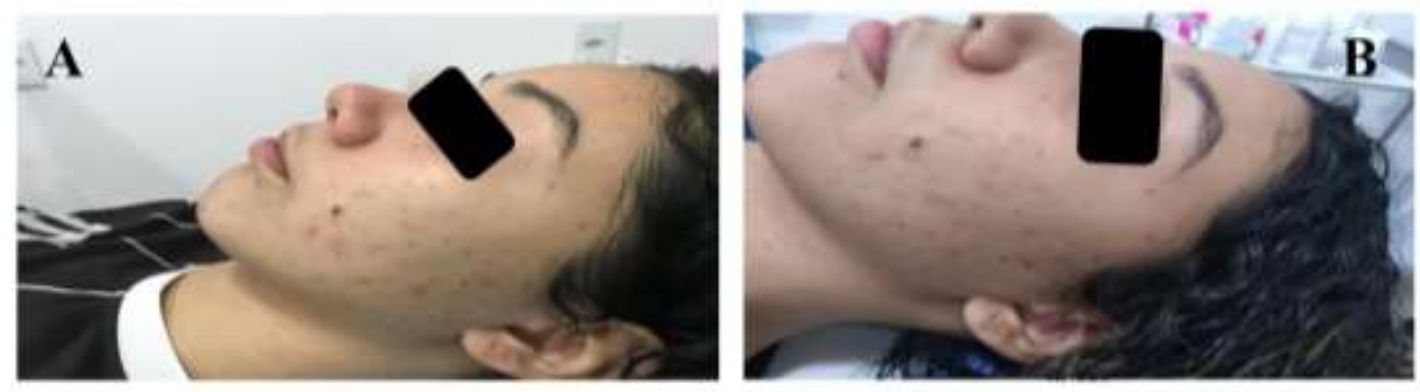

Paciente 1. Grupo 1 - Utilização do gel da camomila a $5 \%$. A. avaliação inicial. B. Avaliação final.

Fonte: Autores.

Figura 2: Paciente 2 do Grupo 2.
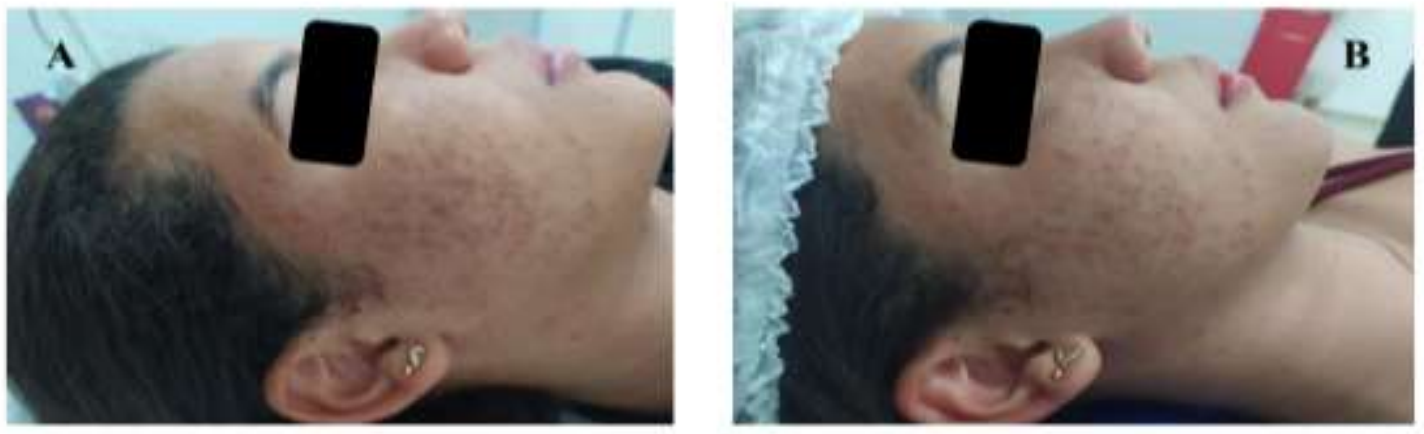

Paciente 2. Grupo 2 - Utilização do LED Vermelho. A. Avaliação inicial. B. Avaliação final 
Figura 3: Paciente 3 do Grupo 3.
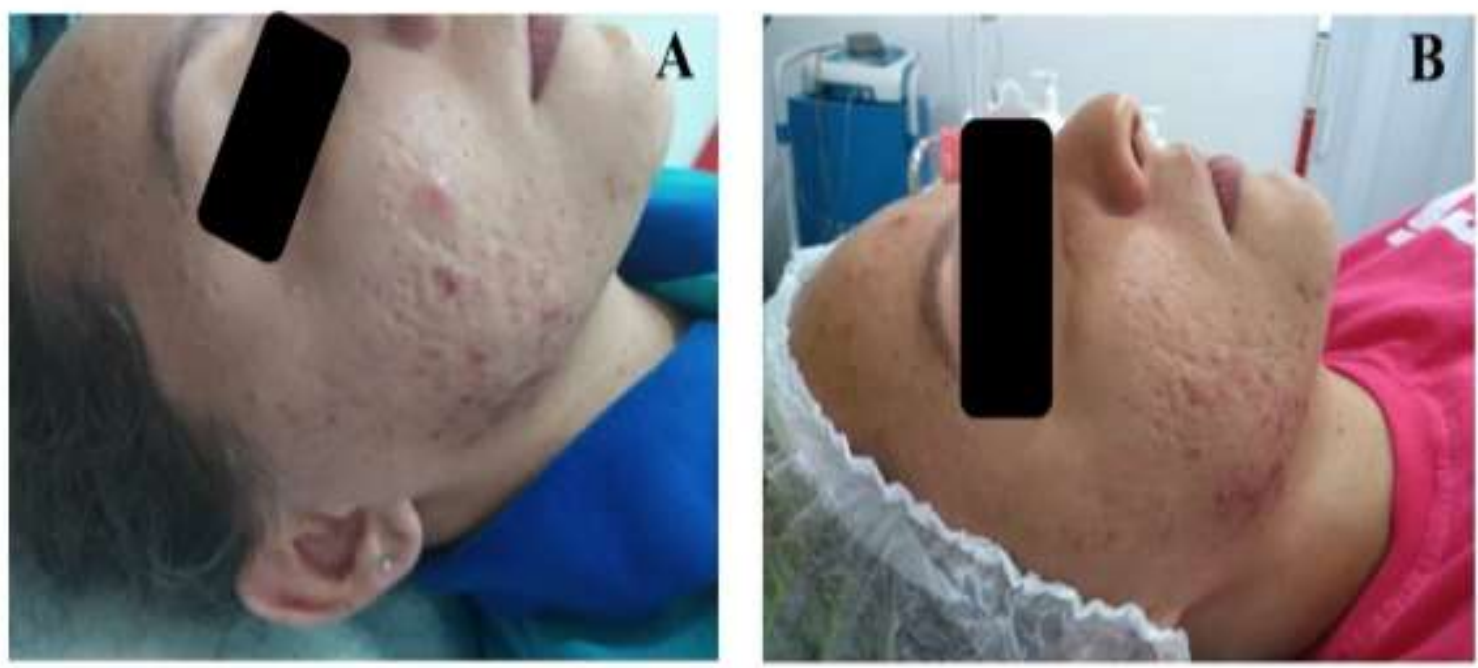

Paciente 3. Grupo 3 - Utilização da Camomila a 5\% + LED Vermelho. A. Avaliação inicial. B. Avaliação final.

Fonte: Autores.

Figura 4: Paciente 4 do Grupo 4.
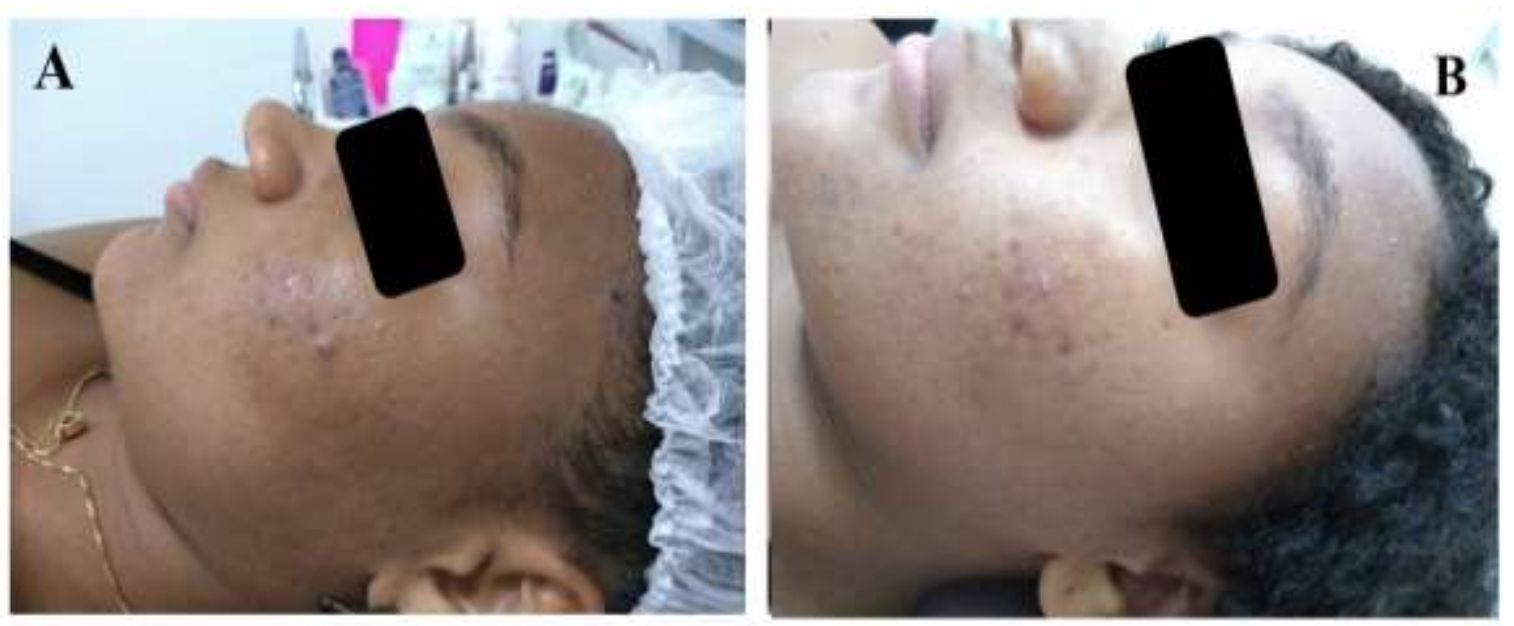

Paciente 4. Grupo 4 - Utilização de loção secativa. A. Avaliação inicial. B. Avaliação final. Fonte: Autores. 
Gráfico 4: Avaliação final do número de lesões das pacientes submetidas ao tratamento com o uso do gel de Camomila 5\%. GRUPO 1 (G1). Floriano-PI, 2021.

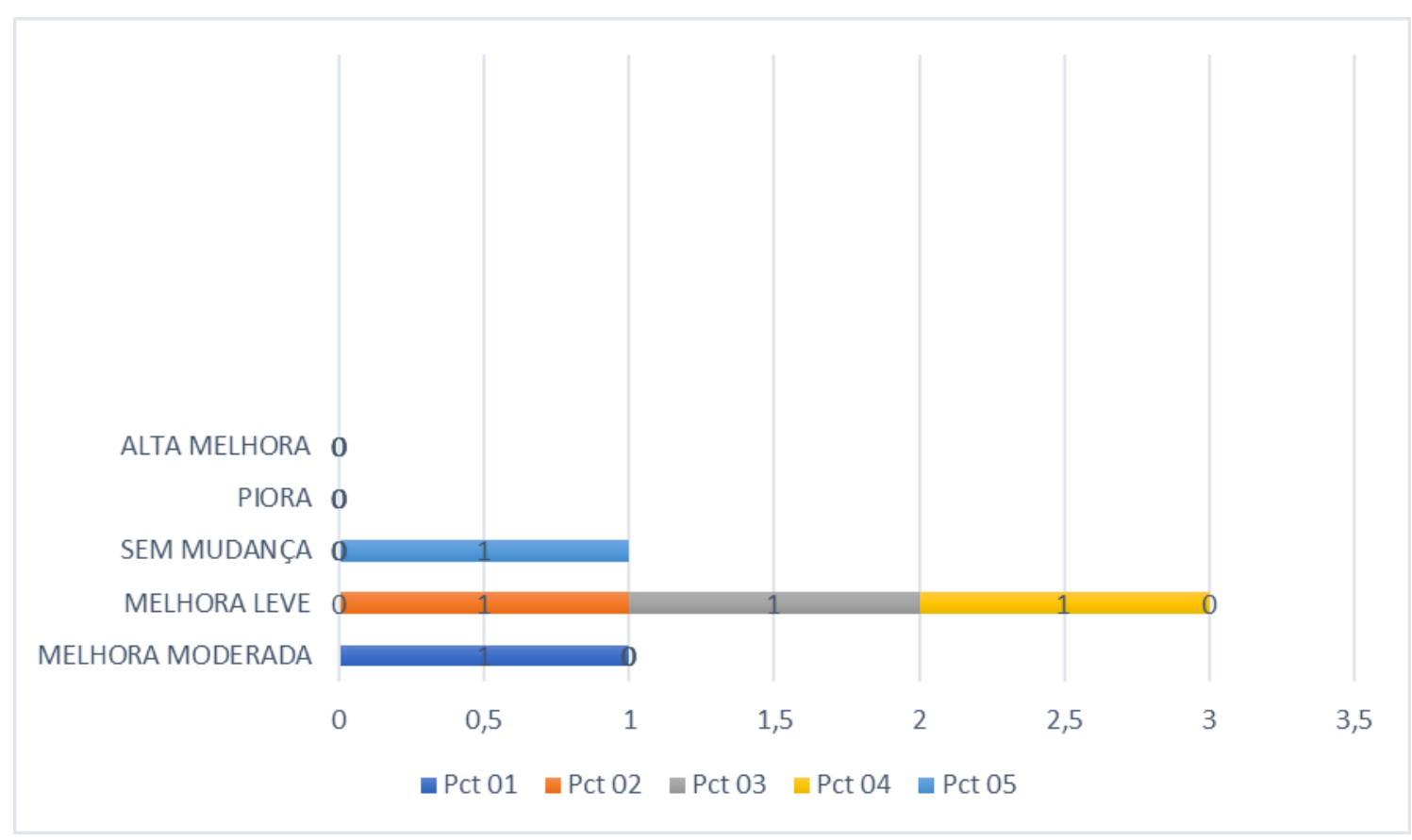

Fonte: Autores.

Quanto ao número de lesões do grupo (G1) que utilizou o gel da Camomila a 5\%, na avaliação final, foram classificadas tres pacientes com melhora leve (60\%), 01 paciente com melhora moderada $(20 \%)$ e 01 paciente que não apresentou mudança (20\%). Comparando-se ao grupo (G3), que fez a associação com o Led vermelho de baixa frequência, no grupo (G1) nenhuma paciente apresentou alta melhora. Analisando os resultados do Grupo (G1) houve melhora leve na inflamação da acne.

Confirmando o autor Lorenzi, 2004 que a atividade predominante do extrato aquoso é espasmolitica, enquanto o extrato alcoólico apresenta uma atividade antiflogística. O camazuleno possui reconhecida atividade anti-inflamatória, que é reforçada pela presença de matricina e alfa bisabolol. 
Gráfico 5: Avaliação final do número de lesões das pacientes submetidas ao tratamento com o uso do LED vermelho de baixa frequência. GRUPO 2 (G2). Floriano-PI, 2021.

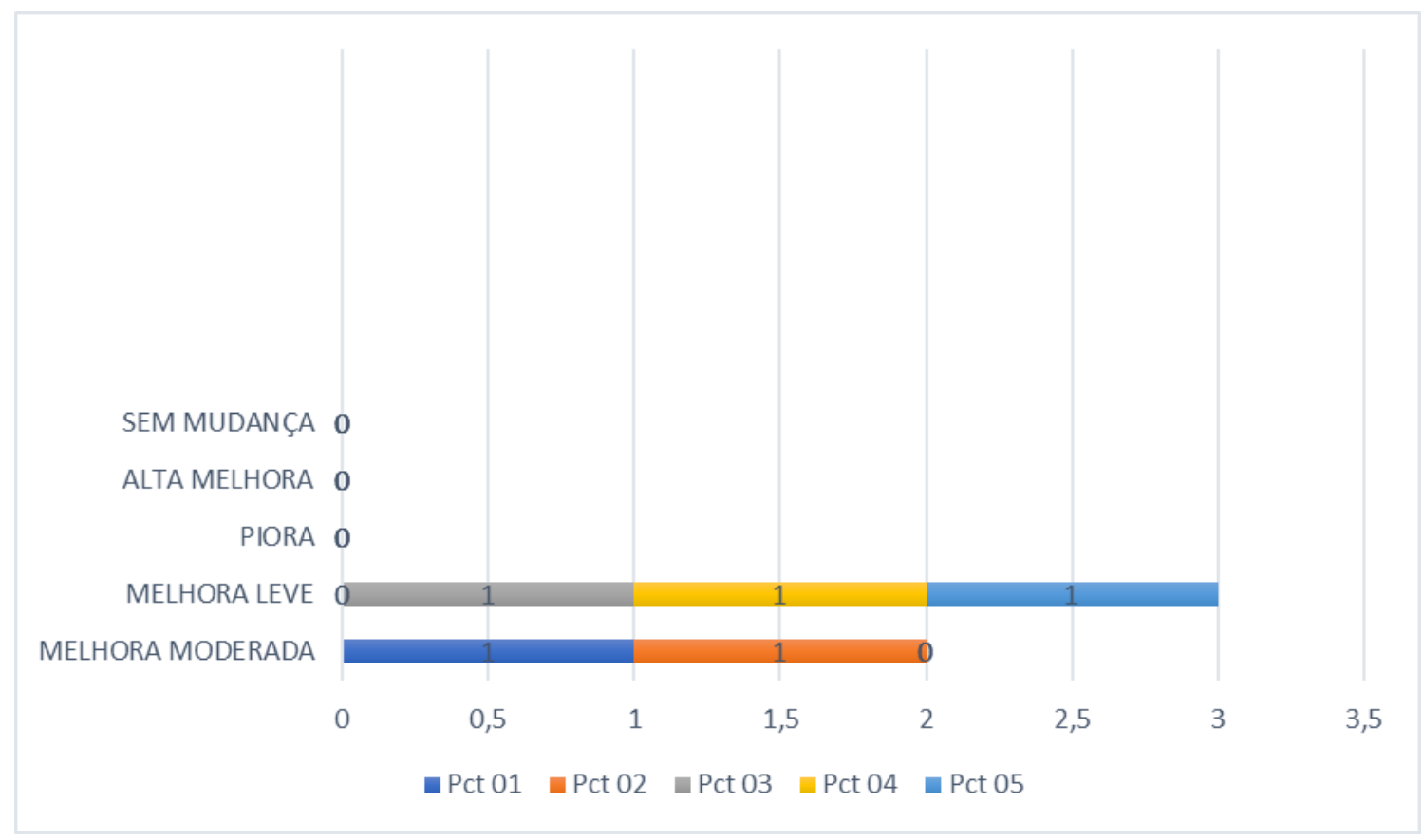

Fonte: Autores.

Ao analisar o Gráfico 5, o grupo (G2) que utilizou o LED Vermelho de baixa frequência, na avaliação final, foram classificadas 02 pacientes com melhora moderada (40\%) e 03 pacientes com melhora leve (60\%). Nesse grupo quanto ao número da contagem de lesões, não foi observado paciente "sem mudança”, porém ao comparar com o grupo (G3) também não apresentou paciente com alta melhora.

Quanto as estimativas do grupo (G2), somente o LED Vermelho não foi suficiente para melhorar o aspecto cutâneo da pele. De acordo com as variáveis da Contagem dos Números de Lesão, as mulheres atendidas nesse grupo não ficaram muito satisfeita com tratamento proposto.

Park et al. 2015 mostraram que a luz LED Vermelha contínua melhora a neovascularização em um modelo de ferida na pele em camundongos. No entanto, ainda há controvérsia sobre a eficácia da luz LED em relação aos lasers. Até o momento, a maioria dos estudos foi realizada com luz no espectro vermelho ou infravermelho. Há evidências crescentes de que comprimentos de onda mais curtos podem apoiar significativamente os processos de regeneração de tecidos. 
Gráfico 6: Avaliação final do número de lesões das pacientes submetidas ao tratamento com o uso do gel a 5\% da camomila (matricaria chamomilla l.) associado ao LED Vermelho de baixa frequência. GRUPO 3 (G3). Floriano-PI, 2021.

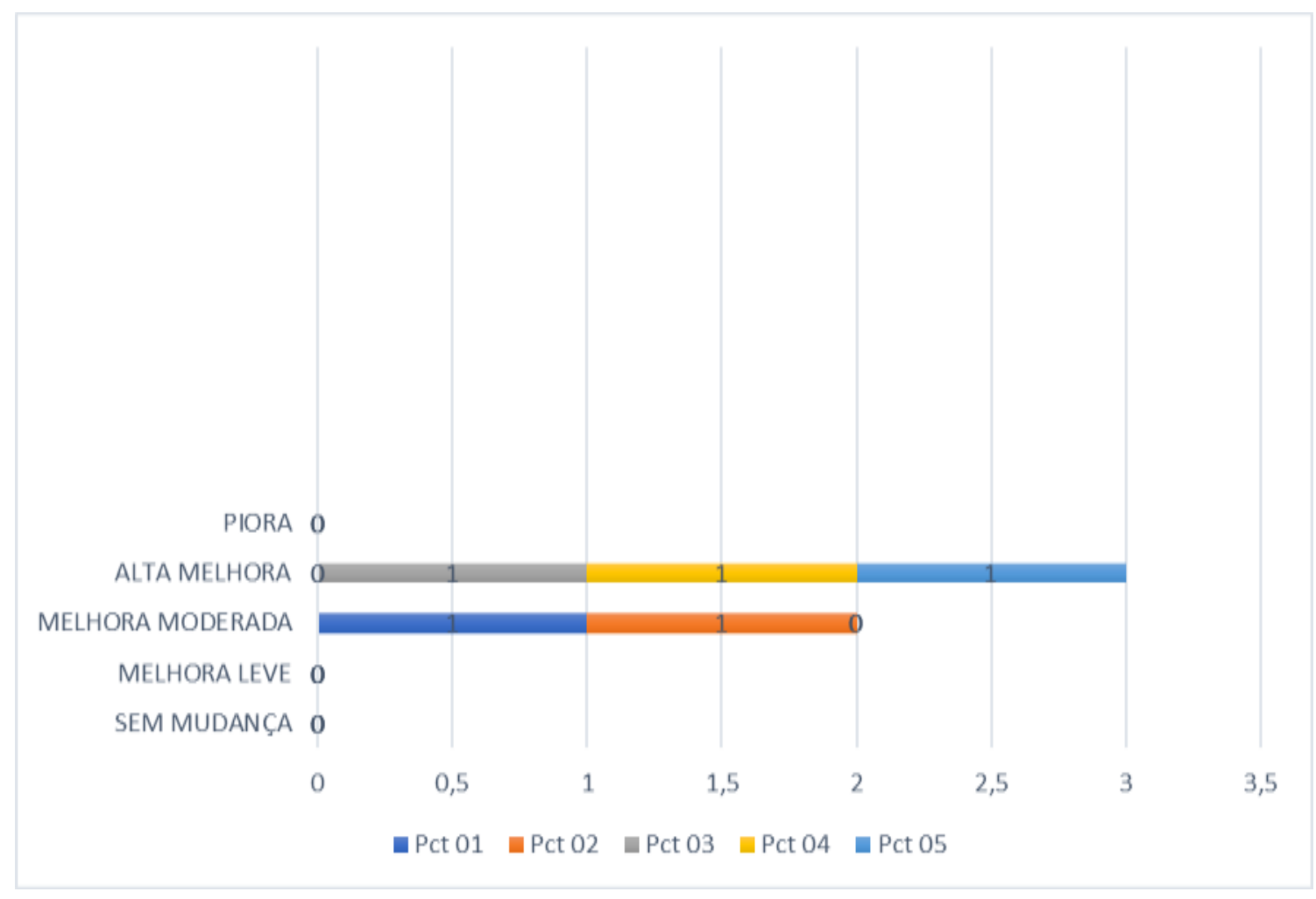

Fonte: Autores.

No Gráfico 6, o grupo (G3) que utilizou o gel a 5\% da camomila (matricaria chamomilla 1.) associado ao aparelho de LED Vermelho de baixa frequência, na avaliação final foram classificadas: duas pacientes com melhora moderada (40\%) e três pacientes com alta melhora $(60 \%)$. Não tiveram pacientes com piora, sem mudanças e melhora leve. Esse foi o grupo que apresentou os resultados mais positivos de acordo com a classificação do número de lesão. Na avaliação final as mulheres atendidas já relatavam uma melhora. Ao unificar esses dois tratamentos (gel da camomila e o LED Vermelho) nas cinco pacientes deste grupo, observou-se uma diminuição da inflamação das glândulas sebáceas e dos folículos pilossebáceos, que formam a acne, o que trouxe resultados satisfatórios no período de avaliação.

Observou-se no experimento pelo grupo (G3) que utilizou o gel a 5\% da camomila associado ao LED Vermelho comparado ao tratamento padrão grupo (G4) da clínica que o número de exposições ao LED Vermelho durante os quatro dias consecutivos contribuíram para o reparo da pele com acne ativa, superando o tratamento padrão de rotina que não era realizado para o tratamento da acne. Sendo assim, uma alternativa para ser incorporada nesse local de atendimento. Confirmando as observações de Na Ji e Suh Dh (2007) ao realizarem um ensaio clínico randomizado, controlado e cego, com 28 voluntários, utilizando apenas o led vermelho em uma hemi face como experimento e outra como controle, com frequência de tratamento de duas vezes por dia, durante oito semanas, por 15 minutos, totalizando 112 sessões, com uma dose cumulativa final 604,8 $\mathrm{J} / \mathrm{cm}^{2}$. Ao realizar a comparação entre hemi face experimento e controle, obteve redução de 55\% do número de lesões quando comparado com o grupo controle (19\%), na $8^{\mathrm{a}}$ semana. Pode-se concluir a efetividade do led vermelho no manejo da acne por si própria, sugerindo que talvez o número de exposições ao tratamento seja muito mais importante do que a dose empregada para obtenção de um resultado positivo.

O extrato da camomila (matricaria chamomilla l.) pode ser incorporado em diversas formas farmacêuticas como géis, loções e sabonetes; em concentrações que variam de 2 a 5\%, sendo de grande valia no tratamento da acne. No experimento ora 
apresentado foi utilizado o gel da camomila a $5 \%$ baseando-se nesses dados farmacológicos, a fim de alcançar uma diminuição da inflamação das pacientes com acne e melhorando assim o aspecto cutâneo da pele.

Observou-se nesse experimento que o grupo (G3) que utilizou o gel a 5\% da camomila associado ao LED Vermelho comparado ao tratamento padrão grupo (G4) da clínica que o número de exposições ao LED Vermelho durante os quatro dias consecutivos contribuíram para o reparo da pele com acne ativa, superando o tratamento padrão de rotina que não era realizado para o tratamento da acne. Sendo assim, uma alternativa para ser incorporada nesse local de atendimento.

Durante a busca de dados para essa pesquisa, estudos foram encontrados sobre o LED Azul, porém vale ressaltar que a escolha pelo LED Vermelho foi para acelerar o processo de cicatrização. Analisando esse grupo (G3) constatou-se o resultado esperado que foi a diminuição da inflamação das lesões da acne. Nesse grupo assim como os grupos (G1-G2-G4), as mulheres atendidas não apresentaram outros sinais da inflamação, nenhuma ardência e nem outro incomodo com a associação do gel e do LED ao final do 04 atendimento. Sendo necessário a continuidade das aplicações e os cuidados diário com a pele. As mudanças de hábitos e a procura por um profissional especializado são importantes.

Gráfico 7: Avaliação final do número de lesões das pacientes submetidas ao tratamento padrão para acne vulgar. GRUPO 4 (G4). Floriano-PI, 2021.

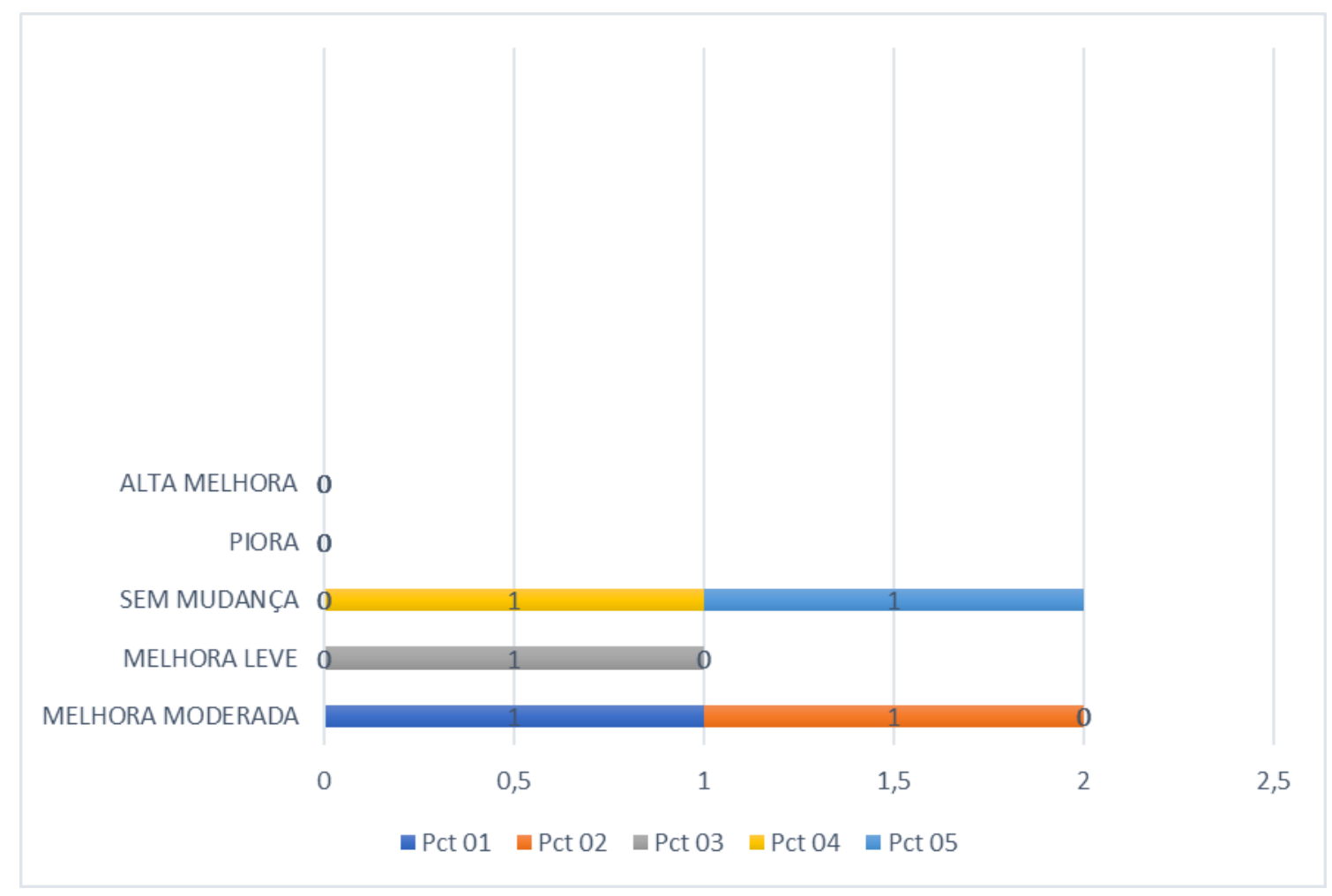

Fonte: Autores.

Quanto ao número de lesões do grupo (G4), grupo padrão (uso de uma loção secativa a base de acneol + ácido lactobiônico + TGP2 peptídeo) que utilizou a loção secativa, tratamento de rotina da clínica, na avaliação final foram classificadas duas pacientes com melhora moderada (40\%), uma paciente com melhora leve (20\%) e duas pacientes sem mudança (40\%).

Ao comparar os resultados do grupo (G4) (grupo padrão) com o grupo (G3) (camomila + LED Vermelho) observou-se que as pacientes do grupo (G4) não apresentaram mudanças significativas do grau da acne como observado no grupo atendido com o gel a 5\% de camomila e o LED Vermelho, mesmo compreendendo que uma loção secativa pode 
atenuar a oleosidade. O diferencial do grupo (G3) foi aplicação do LED Vermelho que diminui a inflamação e o gel a 5\% da camomila que teve efeito calmante na pele de forma associada.

Quanto a análise desse grupo (G4) é importante salientar que os cosméticos irão sim interferir no tratamento da acne, mas a utilização do LED Vermelho pode influenciar mais ainda os resultados. Esse grupo que não utilizou o LED não obteve mudanças quanto ao número de lesão, por isso a importância de associar o LED Vermelho no tratamento da acne. Comparando os resultados com os demais grupos, esse grupo também não teve mulheres que apresentassem variável de alta melhora de acordo com a classificação da Contagem do número de lesão.

\section{Considerações Finais}

O tratamento da acne com uso do gel da camomila a 5\% associada ao aparelho de LED Vermelho de baixa frequência $(4,82-3,84 \mathrm{~Hz})$, mostrou-se efetivo na diminuição do processo inflamatório da pele. Uma terapêutica segura, acessível e eficaz. Sendo também importante outros cuidados especiais com a pele nos pacientes com essa afecção dermatológica. Portanto, trabalhos referentes a esse assunto devem ser estimulados, pois não existe ainda uma conduta uniforme para o tratamento de pacientes com Acne Vulgar.

\section{Referências}

Alonso, J. (2004). Tratado de fitofármacos y nutracéuticos. ED. CORPUS.

Carroll et al. (2014). Developments in low level light therapy (LLLT) for dentistry. Dent Mater. 30(5) :465-75.

Dungel, et al. (2014). A terapia de luz de baixo nível por LED de diferentes comprimentos de onda induz a angiogênese e melhora a cicatrização de feridas isquêmicas. Lasers Surg. Med.46, 773-780

Gardiner. (2007). Medicina complementar, holística e integrativa: Camomila. Pediatr. Rev. 28, 16-18.

Hernandéz, D. B. (2018). Cicatrização clínica de feridas e estomaterapia aplicada. Enfermagem em foco Editora.

Lorenzi, H. (2004). Plantas Medicinais no Brasil: nativas e exóticas.

Manual MSD: MERCK SHARP E DOHME (MSD). Versão Para Profissional Da Saúde. https://www.msdmanuals.com/pt-br/profissional.

Mcginley, et al. apud Matarazzo, (2016). Regional variations in density of cutaneous Propionibacteria: correlation of Propionibacterium acnes populations with sebaceous secretion. J Clin Microbiol. 12:672-5.

Mckay, D. L.; \& Blumberg, J. B. (2006). A review of the bioactivity and potential health benefits of chamomile tea (Matricaria Recutita L.). Phytotherapy Research, 20(7), 519-530.

Michalun, N.; \& Michalun, M. V. Dicionário de ingredientes para Cosmética e cuidados da pele.: Cengage learning; SENAC, 2010.

Na, J. I., \& Suh, D. H. (2017). Red Light Phototherapy Alone Is Effective for Acne Vulgaris: Randomized, Single-Blinded Clinical Trial. Dermatol Surg. 33()$: 1228-33$.

Oliveira, A. L., et al. (2014). Curso Didático de Estética 2 (2a ed.). Yendis.

PARK. Aglomerado de células estromais derivadas do adiposo com terapia de luz aumenta a angiogênese e a cicatrização de feridas cutâneas em camundongos.Biochem. Biophys. Res. Comum.462, 171-177.

Peplow et al. (2010). Laser photobiomodulation of proliferation of cells in culture: a review of human and animal studies. Photomed Laser Surg 28(Suppl. 1): S3-40

Rebello, T. (2005). Guia de Produtos Cosméticos. (6a ed), SENAC.

Ribeiro, C. J. (2010). Cosmetologia Aplicada a Dermoestética. (2a ed), Pharmabooks Editora,

Silveira A. A. (2018). Ações dermatológicas no cuidado com a pele oleosa. In: Ornazzano G, organizadora. Questões de arteterapia.

Sociedade Brasileira de Dermatologia. (2018). (4a ed.). Artes Médicas.

Yamada \& Silva. (2017). Uso do Led para o Tratamento da Acne. 